\title{
Denoising of Document Images using Discrete Curvelet Transform for OCR Applications
}

\author{
C. Patvardhan \\ Electrical Engg. Department \\ Dayalbagh Educational Institute \\ Dayalbagh, Agra, UP, India
}

\author{
A. K. Verma \\ Electrical Engg. Department \\ Dayalbagh Educational Institute \\ Dayalbagh, Agra, UP, India
}

\author{
C. V. Lakshmi \\ Physics \& CS Department \\ Dayalbagh Educational Institute \\ Dayalbagh, Agra, UP, India
}

\begin{abstract}
In this paper, a denoising and binarization scheme of document images corrupted by white Gaussian noise and Impulse noise is presented using Curvelet Transform. The ability of sparse representation and edge preservation of Curvelet transform is utilized. Impulse noise gets added during document scanning or after binarization of scanned document images. White Gaussian noise corrupts the document images during transmission. The presence of either type of noise or a combination of them can severely degrade the performance of any OCR system. In the proposed denoising scheme, the curvelet transform is used with level dependent threshold calculated by modified sqtwolog method (universal threshold) at each scale with estimation of noise standard deviation. The noisy curvelet coefficients are thresholded by Hard Thresholding method. After curvelet based denoising, the image is binarized using global Otsu method and post processed to smoothen the text boundaries and remove isolated pixels for better OCR performance. The curvelet based scheme is compared with a wavelet transform based scheme and a modified wavelet based scheme with edge preservation. The results show that curvelet based scheme performs better in case of images containing Gaussian, Impulse and a combination of both the noises.
\end{abstract}

\section{General Terms}

Document Image Processing, OCR

\section{Keywords}

Curvelets, Wavelets, Edge Preservation, Gaussian Noise, Impulse Noise, Thresholding, Denoising, Document Images

\section{INTRODUCTION}

Digital Images are generally corrupted by noise during the process of acquisition and transmission. This noise degrades the quality of digital image. Consequently the performance of any system manipulating these images is also degraded. Specifically in the context of images of documents, Impulse noise is added during scanning or binarization for OCR (Optical Character Recognition) applications. This produces several tiny dots scattered due to uneven grayscale intensities. This may cause false character recognition. Also during transmission of digital images, Gaussian noise degrades the document image quality and causes poor recognition during use of the OCR. When SNR is very low, the small text font may almost dissolve in the noise and hence cannot be recognized properly. Even a document image corrupted by only Gaussian noise, when binarized, exhibits Impulse noise. Therefore, removal of noise in document images corrupted by Gaussian and Impulse noises before OCR is important to ensure accuracy of character recognition.
The noise removal techniques can mainly be divided into two groups: Spatial domain and Frequency domain based techniques. The spatial domain approaches [1] mainly smoothen the images to reduce noise and therefore, some important image properties such as edges are also blurred. Particularly for text images, where the information lies in the edges, blurred edges cause poor recognition. Some spatial domain techniques are given in $[2,3,4,5]$. A feature preserving with edge enhancement scheme for ultrasonic images is presented in [2]. In this scheme, denoising is performed by anisotropic diffusion and edge enhancement is done by hyperbolic tangent function. In [3], a method of image denoising based on principal component analysis is presented. This method works on local pixel grouping used for PCA training. In [4], a Total Variation (TV) algorithm for images is presented and in [5], its application to image denoising is proposed. The total variation of the image is minimized subject to constraints involving the statistics of the noise. The constraints are imposed using Lagrange multipliers and the solution is obtained using the gradient-projection. The transform domain based denoising methods particularly those based on Wavelet transform are more popular. An adaptive edge preserving image denoising method based on wavelet transform is proposed in [6]. In this scheme, edge and nonedge coefficients are identified. Then non-edge coefficients are thresholded more as compared to edge coefficients to preserve edge features. A despeckling method for SAR images with edge enhancement based on Stationary Wavelet Transform (SWT) is given in [7]. Here, Bayesian wavelet shrinkage is used for despeckling and modified ratio edge detector is used to obtain edge information. Another method based on adaptive threshold estimation considering generalized Gaussian distribution of wavelet coefficients is given in [8]. This method finds level dependent threshold based on some statistical properties of wavelet coefficients i.e. standard deviation, arithmetic mean and geometric mean.

A large number of wavelet based image denoising methods along with several types of thresholding have been proposed in recent years. These methods are mainly reported for images such as Lena, Barbara, Boat etc. The general methods based on wavelet transform using soft thresholding are not capable of preserving edges and fine details and therefore, are not suitable for denoising text images for OCR applications. The hard thresholding does better than soft thresholding for text images but at higher noise level this results in broken characters with irregular text edges. Curvelet transform based denoising is expected to be better in terms of curvature and edge preservation in digital images. An elementary work of denoising based on the curvelet transform is proposed in [9]. In this paper, a simple denoising scheme is implemented where curvelet coefficients are hard threshloded. The proposed scheme is compared with several wavelet based 
schemes and its superiority of preserving curves and edges is highlighted. A curvelet based method which uses modified threshold estimation is given in [10]. In this paper, curvelet coefficients are thresholded using an adaptively calculated threshold which depends on scale and local noise variance giving improved performance with details preservation. Another method for removing edge noise (speckles) in bilevel drawing images based on curvelet transform is proposed in [11]. This method shows the superiority of curvelet transform in edges and curve preservation. A method based on curvelet shrinkage and nonlinear anisotropic diffusion is presented in [12]. This method is able to suppress the pseudoGibbs and curvelet like artifacts in denoised image. A context adaptive denoising method based on curvelet coefficients statistics modeling is proposed in [13]. This method works by distinguishing two classes of curvelet coefficients, one which is free of significant noise and the other has noise components. This method uses Probshrink method to threshold curvelet coefficients.

In this proposed work, wavelet transform based denoising schemes and a curvelet transform based scheme for denoising of document images which are contaminated by white Gaussian noise and Impulse noise are presented and compared. The rest of the paper is divided in the various sections. Section 2 briefly explains Curvelet transform theory. Section 3 presents the proposed denoising schemes. Section 4 gives experimental results and analysis. Section 5 gives some conclusions followed by section 6 of references.

\section{CURVELET TRANSFORM}

Wavelet transform is widely used for denoising but it suffers from shift and rotation sensitivity as well as shows poor directionality. Curvelet transform is more suitable for detection of directional properties as it provides optimally sparse representation of objects giving maximum energy concentration along the edges.The theory of Curvelet transform presented here is directly inspired by the elegant work presented in [9]. Curvelets are the basis elements which show high directional sensitivity and are highly anisotropic. Curvelets have variable width and variable length and so a variable anisotropy. The length and width at fine scales are related by a scaling law (width $\approx$ lengt $^{2}$ ) and so the anisotropy increases with decreasing scale like a power law. In two dimensional plane, Curvelets are localized not only in position (the spatial domain) and scale (the frequency domain), but also in orientation. The Curvelet transform, like the wavelet transform, is a multiscale transform. In addition, the curvelet transform is based on a certain anisotropic scaling principle which is quite different from the isotropic scaling of wavelets. The discrete curvelet transform of 2-D function $f\left(x_{1}, x_{2}\right)$ makes use of dyadic sequence of scales and a bank of filters $\left(P_{0} f, \Delta_{1} f, \Delta_{2} f, \ldots \ldots\right)$ with the property that the bandpass filter $\Delta_{s}$ is concentrated near the frequencies $\left[2^{2 s}, 2^{2 s+2}\right], \quad$ e.g. $\quad \Delta_{s}=\psi_{2 s} * f, \widehat{\psi_{2 s}}(\xi)=$ $\hat{\psi}\left(2^{-2 s} \xi\right)$. In wavelet theory, one uses decomposition into dyadic sub-bands $\left[2^{s}, 2^{s+1}\right]$, In contrast, the sub-bands used in the discrete curvelet transform of continuum function have the nonstandard form $\left[2^{2 s}, 2^{2 s+2}\right]$. The basic process of the digital realization for curvelet transform of a 2-D function $f\left(x_{1}, x_{2}\right)$ is given as follows.

1)Sub-band Decomposition: The function $f\left(x_{1}, x_{2}\right)$ is decomposed into sub-bands using 'a trous' algorithm as,

$$
f \rightarrow\left(P_{0} f, \Delta_{1} f, \Delta_{2} f, \ldots . .\right)
$$

The different sub-bands $\Delta_{s} f$ contain details about $2^{-2 s}$ wide.
2) Smooth Partitioning: Each sub-band is smoothly windowed into squares of an appropriate scale (of side length $\left.\sim 2^{-s}\right), \Delta_{S} f \rightarrow\left(w_{Q} \Delta_{S} f\right)_{Q \in Q_{S}}$

Where, $w_{Q}$ is a collection of smooth window localized around dyadic squares $Q=\left[k_{1} / 2^{s},\left(k_{1}+1\right) / 2^{s}\right] \times[k 2 /$ $\left.2^{s},\left(k_{2}+1\right) / 2^{s}\right]$

3) Renormalization: Each resulting square is renormalized to unit scale,

$$
g_{Q}=\left(T_{Q}\right)^{-1}\left(w_{Q} \Delta_{s} f\right), \quad Q \in Q_{S}
$$

Where, $\left(T_{Q} f\right)\left(x_{1}, x_{2}\right)=2^{s} f\left(2^{S} x_{1}-k_{1}, 2^{s} x_{2}-k_{2}\right)$ is a renormalization operator.

4) Ridgelet Analysis: Each square is analyzed via the orthonormal discrete ridgelet transform. This is a system of basis elements $p_{\lambda}$ making an orthonormal basis for $L^{2}\left(R^{2}\right): \alpha_{\mu}=\left\langle g_{Q}, p_{\lambda}\right\rangle$.In this definition, the two dyadic sub-bands $\left[2^{2 s}, 2^{2 s+1}\right]$ and $\left[2^{2 s+1}, 2^{2 s+2}\right]$ are merged before applying ridgelet transform.

\section{DENOISING SCHEME}

Let the original image (2D signal) be represented by $I(i, j)$. The noisy Image $I_{n}(i, j)$ is given by, $I_{n}(i, j)=$ $I(i, j)+\sigma \cdot z_{g}(i, j)$, Where, $\sigma$ is the noise standard deviation and $z_{g}(i, j)$ is the white noise of zero mean $\left(\mu_{g}=0\right)$ and unit variance $\left(\sigma_{g}^{2}=1\right)$. Here the objective is to obtain the best estimate $I_{d}(i, j)$ of noisy image $I_{n}(i, j)$ and binarize the denoised image $I_{d}(i, j)$ to achieve its binary version $I_{d b i n}(i, j)$ for OCR application with maximum character quality preservation. In this paper, three methods are implemented to denoise images corrupted by white Gaussian noise and compared. The first method is a wavelet based denoising scheme. The Second method is also a wavelet based denosing scheme motivated by work done in [6], with different thresholding of edge and non-edge components to preserve the text edges and the third scheme is based on Curvelet Transform with morphological operation. These methods are now presented.

\subsection{Wavelet Based Scheme (Method 1)}

The wavelet based scheme for denoising is shown in figure 1.The scheme has following main steps,

a) Find 2 - Level wavelet transform of noisy image $I_{n}(i, j)$.

b) Calculate noise standard deviation $(\sigma)$ and estimate threshold $(\lambda)$ for each level.

c) Perform hard thresholding of wavelet coefficients at each level of decomposition.

d) Perform 2 - Level inverse wavelet transform of thresholded wavelet coefficients to get denoised image $I_{d}(i, j)$.

e) Obtain binary image $I_{d b i n}(i, j)$ of denoised image $I_{d}(i, j)$ by global Otsu method.

f) Find the Evaluation Parameters between binary version of original noise free image and $I_{d b i n}(i, j)$ to observe the quality of denoising.

For wavelet transform decomposition, Haar wavelet is used due to its discrete nature which does not blur the image, while higher order smooth wavelets create blur in the images consequently, result in artifacts in the binarized image. Also, hard thresholding is used because soft thresholding removes some edge coefficients creating smoothness (blur) on the text edges which is not desirable in this case. The threshold $(\lambda)$ used is the Universal Threshold [14] for thresholding, which is given as, 


$$
\lambda_{j}=\sigma \sqrt{2 \log \left(N_{j}\right)}
$$

Where, $N_{j}$ is the size of the wavelet coefficient matrix at $j^{\text {th }}$ level and $\sigma$ is the noise standard deviation. The value of noise standard deviation can be calculated by Median Absolute Deviation (MAD) of high frequency wavelet coefficients $(\mathrm{cH}, \mathrm{cV}$ and $\mathrm{cD}$, in case of an image) of noisy image at level 1 of decomposition. It is given as,

$$
\sigma=\frac{M A D}{0.6745}=\frac{\text { median }\left(\left|\omega_{k}\right|\right)}{0.6745}
$$

Where, $\omega_{k}$ represent wavelet coefficients at scale 1 [14]. In the proposed scheme, the input noisy image is decomposed in 2 levels and the threshold for each level is found then wavelet coefficients are hard thresholded to get the denoised document image.

\subsection{Wavelet based scheme with Edge Preservation (Method 2)}

In the method of section 3.1, the simple wavelet based denoising scheme is mentioned. This scheme also thresholds the edges of text and therefore, results in broken text and text with irregular boundaries when the denoised image is binarized. To enhance the edge details, a modified version of work proposed in [6], which is based on wavelet shrinkage is shown in figure 2.Followings are the steps of the scheme of figure 2,

a) Find $2-$ Level wavelet transform of noisy image $I_{n}(i, j)$.

b) Find Edge Maps for each level by approximation coefficients.

c) Calculate noise standard deviation $(\sigma)$ and estimate threshold $(\lambda)$ for each level

d) Perform hard thresholding of non-edge wavelet coefficients by $\lambda_{\text {nonedge }}$ and of edge coefficients by $\lambda_{\text {edge }}$ at each level of decomposition. $\left(\lambda_{\text {edge }}=\right.$ $0.3 \lambda_{\text {nonedge }}=0.3 \lambda$ ).

e) Perform 2 - Level inverse wavelet transform of thresholded wavelet coefficients to get denoised image $I_{d}(i, j)$.

f) Obtain binary image $I_{d b i n}(i, j)$ of denoised image $I_{d}(i, j)$ by global Otsu method.

g) Find the Evaluation Parametersbetween binary version of original noise free image and $I_{d b i n}(i, j)$ to observe the quality of denoising.
In this proposed scheme, the edge and non-edge coefficients are identified with help of an edge map. To preserve text edges, the edge coefficients are less thresholded as compared to non-edge coefficients.

The edge map is a binary image (' 1 ' represent edges and ' 0 ' represent non-edges) obtained from approximation coefficients (cA) by applying Canny edge detection.

The reason why edge maps are obtained from low frequency approximation coefficients is that,approximation coefficients are less affected by Gaussian noise as major part of noise is absorbed in detail coefficients. Thus edge maps obtained represent correct edges of text even in the presence of Gaussian noise however, this is only possible up to few levels of decomposition. Beyond $2^{\text {nd }}$ level almost all edges will vanish in approximation coefficients. Therefore, the edge map cannot be found correctly at higher levels. Also, in case of very high noise, these edge maps cannot be determined properly. These are some of the limitations of this proposed scheme.The thresholds for edge and non-edge coefficients are found by universal threshold method (eqn. 1) for each level as discussed in section 3.1. It is found empirically after testing several images that edge coefficient threshold should be kept $30 \%$ of non-edge coefficient i.e. $\lambda_{\text {edge }}=0.3 \lambda_{\text {nonedge }}$. The hard thresholding is used for denoising in this case also.

\subsection{Curvelet based Scheme (Method 3)}

The method discussed in section 3.2 works well for low noise as its performance depends on how correctly the edge maps are found. As the noise increases, edge maps are also affected such that the method results in broken characters and irregular character boundaries. To achieve smooth characters, even in presence of large noise, the curvelet transform based approach is proposed and has shown better results. This approach is presented in figure 3 . The denoising scheme shown in figure 3 has the following steps,

a) Find curvelet coefficients of input noisy image $I_{n}(i, j)$ of size $N \times N$ up to scale $L$, which is given by $L=$ ceil $(\log 2(N)-3)$. If input image is of size $M \times$ $N$ then $L=\operatorname{ceil}(\log 2(\min (M, N))-3)$.

b) Calculate noise standard deviation $(\sigma)$ and estimate threshold $(\lambda)$ for each scale.

c) Perform hard thresholding of curvelet coefficients.

d) Perform inverse curvelet transform to get the denoised image $I_{d}(i, j)$.

e) Binarize the denoised image by global Otsu and obtain binary image $I_{d b i n}(i, j)$.

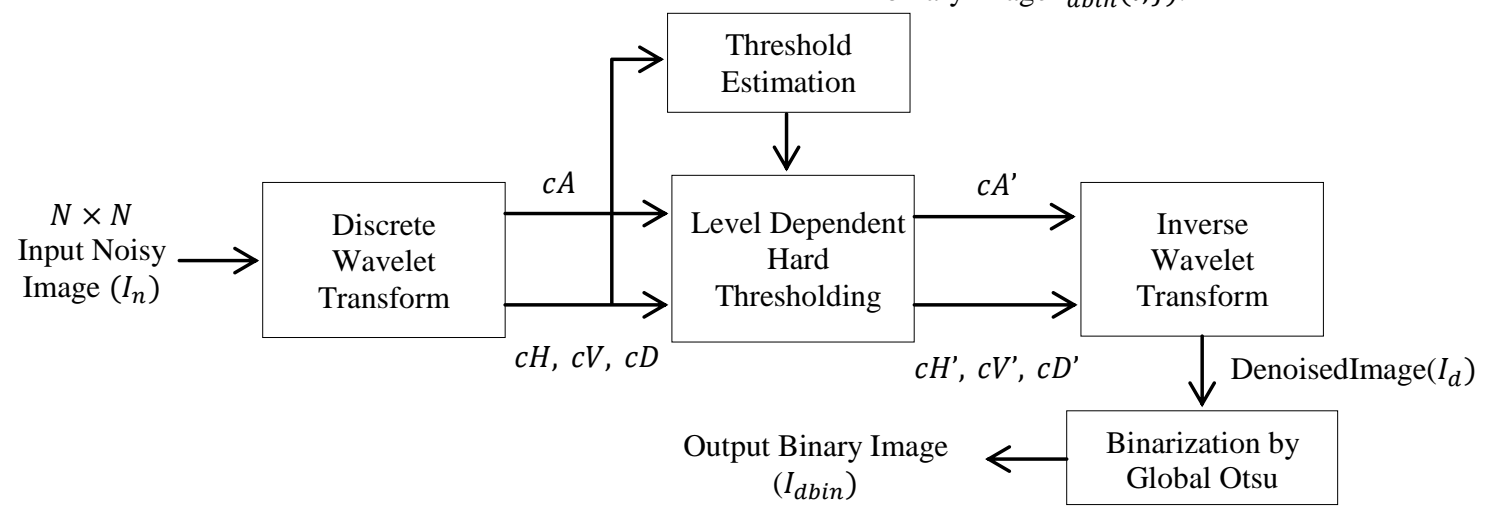

Figure 1: Wavelet based Denoising Scheme 


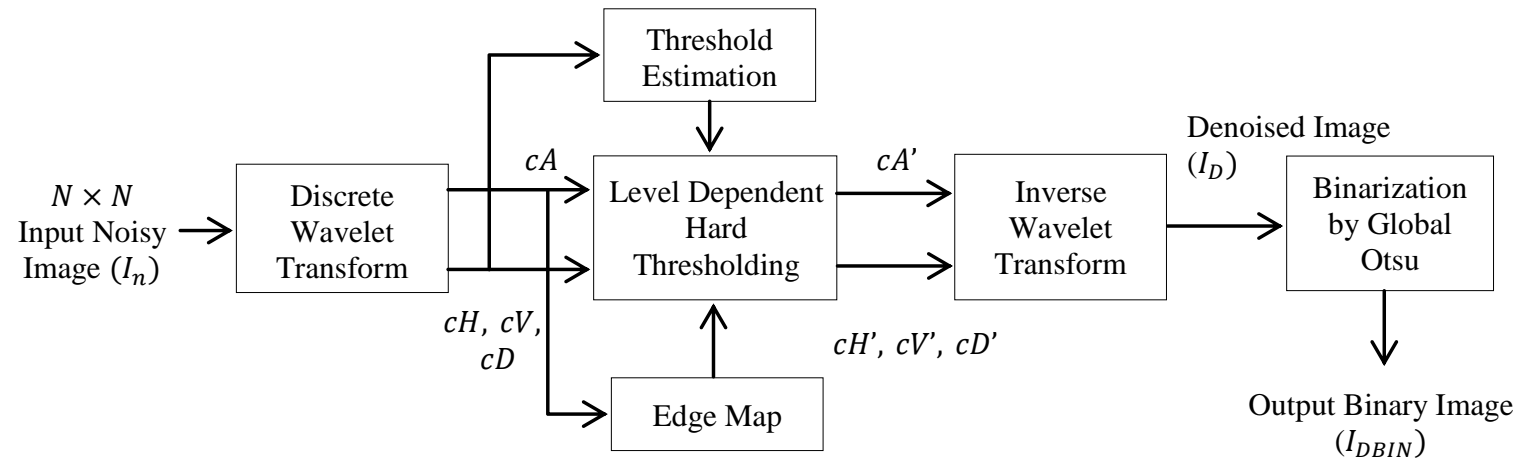

Figure 2: Wavelet based Denoising Scheme with Edge Preservation

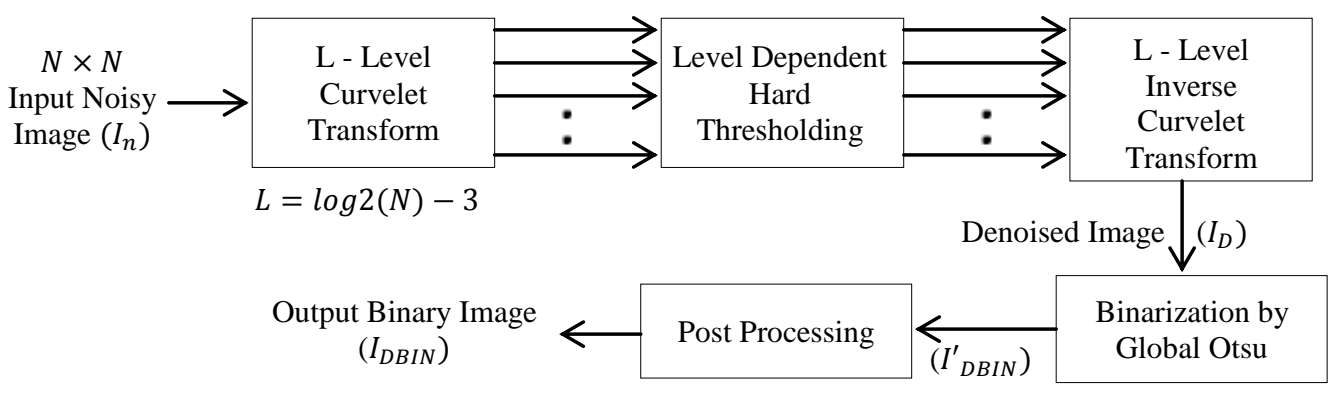

Figure 3: Curvelet Transform based Denoising Scheme with post processing

f) Perform the post processing operations on $I_{\text {dbin }}(i, j)$ to smoothen the text in image.

g) Find the Evaluation Parametersbetween binary version of original noise free image and $I_{d b i n}(i, j)$ to observe the quality of denoising.

In the proposed curvelet based scheme, the noise standard deviation $(\sigma)$ is found as in eqn. 2. But the threshold used is the modified form of eqn. 1 and is given as,

$$
\lambda_{j}=K \cdot \sigma \sqrt{2 \log \left(N_{j}\right)}
$$

Where, $N_{j}$ is the size of the curvelet coefficient matrix at $j^{\text {th }}$ scale and $\sigma$ is the noise standard deviation and $K$ is the parameter $(0<K<1)$, adjusted to find better PSNR.

It is observed that Universal threshold value as found by eqn. 1 is higher and cuts significant parts of curvelet coefficients. Therefore, the denoised image obtained does not carry good PSNR and characters in binary images become thicker. Equation 1 is modified as eqn. 3 to achieve betterPSNR and characters quality. To find a suitable value of $K$,several test document images of different sizes and different fonts are taken for experiment.

To judge the characters quality of final binarized image, Normalized Coefficient of Correlation (NC) is considered and found between binary image of original noise free image and binarized denoised image by proposed curvelet based scheme because binary image is used for OCR as primary input. The results of this experiment are shown in figure 4 (a) and (b). From the figure 4 (a) and (b), it is observed that for low as well high value of $K$, the value of both PSNR and NC is poor. Figure 4 (a) shows the maximum value of PSNR (not NC) around $K=0.6$ for a variety of images while figure 4 (b) shows the maximum NC (not PSNR) around $K=0.4$.
Therefore, to achieve a good balance of PSNR and NC, the value of $K$ is selected to be 0.5 (in between 0.4 and 0.6 ).

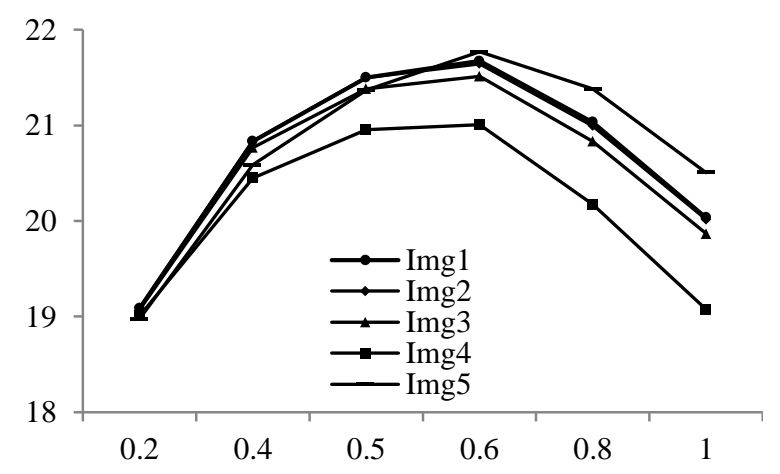

(a)

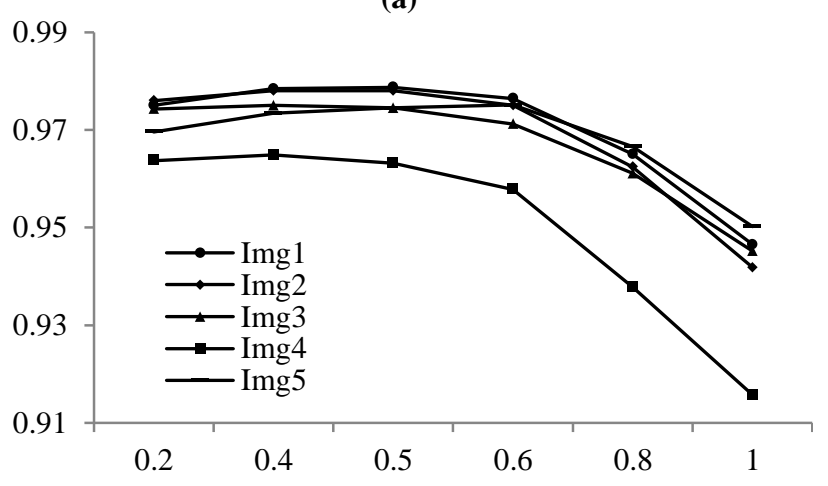

(b)

Figure 4: (a) Variation of PSNR with K, (b) Variation of NC with $K$ 
In the post processing step, a standard morphological operation is performed to remove Spur foreground pixels to smoothen the character edges followed by isolated foreground pixels removal for clean and clear background [1].

\subsection{Strength of Curvelet based method}

The motivation behind the use of curvelet is its strength of optimal representation of edges and curves. It gives sparse representation of edges with few coefficients of high magnitude when aligned. Consider figure 5,

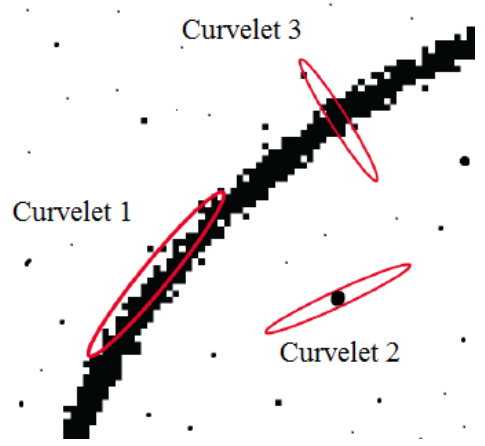

Figure 5: Orientation of Curvelets along a noisy edge

Three different orientations of curveletes near a noisy edge are shown. Here, curvelet 1 is maximally aligned with the edge and therefore, gives larger transform coefficients as compared to curvelet 2 and curvelet 3 . Curvelet 2 is lying on a dot (small pixel group) and gives smaller transform coefficients. If a proper threshold is selected then these coefficients representing small pixel groups can be made zero and therefore in reconstructed image these small pixel groups can be eliminated. Therefore curvelet based approach can also be used in eliminating Impulse type of noise (such as large number of tiny black dots in document images when globally binarized) from the images. If document image is corrupted by both Gaussian and Impulse noise, in such cases, curvelet based approach is expected to perform better with dual advantages of removing both types of noises in the one processing step. The results of denoising of a document image corrupted by only Impulse noise and combination of Gaussian and Impulse are presented in section 4.

\section{EXPERIMENTAL RESULTS AND ANALYSIS}

The normal wavelet based method, wavelet based method with edge preservation which is adapted from [6] and the proposed Curvelet based denoising algorithm are tested on various types of document images of different sizes, font types and font sizes which are corrupted by white Gaussian noise of different strengths.

In this paper the four grayscale document images that are taken for experiments in denoising and binarization are shown in figure 6 .

To compare the binarization quality of all the three methods, some well-known evaluation parameters [15] are used such as (a) F-Measure (FM), (b) Negative RateMetric (NRM),(c) PSNR and (d) Normalized correlation (NC) calculated between binarized input image (Ground Truth image) and output binarized image.

For different noise strength, the performance of proposed schemes is shown and compared in table 1 for the example document image of figure 6 (b).

\section{Binarization can be performed either globally or locally. In global methods, a single threshold is selected for whole image while for local binarization (also called adaptive}

(a) Grayscale $(256 \times 256)$

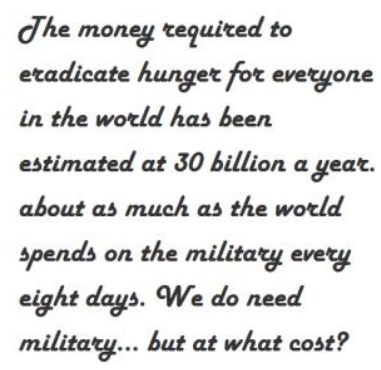

(c) Grayscale (512 × 512)
Washington, May 18 (IANS) Pricing its IPO at $\$ 38$ a share, Facebook, the highly popular social networking site that has over 45 million users in India, is expected to raise $\$ 16$ billion, making it the largest tech IPO in history.

It's reported to be the third largest US IPO ever, trailing only the $\$ 19.7$ billion raised by Visa in March 2008 and the $\$ 18.1$ billion raised by automaker General

(b) Grayscale $(512 \times 512)$

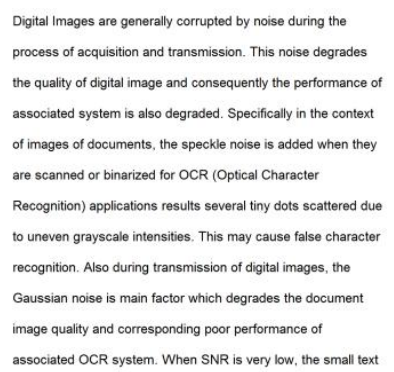

(d) Grayscale (1024 $\times$ 1024)
Figure 6: Various Test Images

Table1: Comparison of all the three methods at different noise strengths for figure 6 (b)

\begin{tabular}{|c|l|c|c|c|c|}
\hline \multicolumn{2}{|c|}{ Sigma $(\boldsymbol{\sigma})$} & $\mathbf{3 0}$ & $\mathbf{4 0}$ & $\mathbf{5 0}$ & $\mathbf{6 0}$ \\
\hline \multirow{3}{*}{ Method } & FM & 94.08 & 89.09 & 84.58 & 81.13 \\
1 & NRM & 0.037 & 0.059 & 0.081 & 0.104 \\
& PSNR & 17.82 & 15.09 & 13.53 & 12.68 \\
& NC & 0.931 & 0.873 & 0.820 & 0.780 \\
\hline \multirow{3}{*}{ Method } & FM & 95.87 & 92.65 & 89.20 & 86.24 \\
2 & NRM & 0.026 & 0.041 & 0.057 & 0.072 \\
& PSNR & 19.39 & 16.84 & 15.12 & 14.03 \\
& NC & 0.952 & 0.914 & 0.874 & 0.840 \\
\hline \multirow{3}{*}{ Method } & FM & 98.17 & 97.47 & 96.63 & 95.72 \\
3 & NRM & 0.012 & 0.014 & 0.017 & 0.021 \\
& PSNR & 22.90 & 21.47 & 20.22 & 19.17 \\
& NC & 0.979 & 0.971 & 0.960 & 0.950 \\
\hline
\end{tabular}

For wavelet based methods Haar wavelet is used and for curvelet based method, the value of $k$ is taken 0.5 as discussed in section 3.3. All the methods use hard thresholding. In case of wavelet based method with edge preservation, the value of threshold and standard deviation of Gaussian filter for Canny edge detection are taken as 0.5 and 0.8 respectively. As can be seen from the table 1 , for low strength of noise, normal wavelet method and method with edge preserve also perform well. As noise strength increases the performance of curvelet based approach becomes much better than the two methods using wavelets. At very high noise level $(\sigma=60)$, the performance of curvelet based approach is better and the quality of output binary image is appropriate for OCR applications. In table 2, the portion of binary image obtained from its denoised document image of figure 6(b) are shown at $\sigma=60$ for all the three methods.

As can been seen from the table 2, the output images of first two methods are degraded severely and are not suitable for correct character recognition while the proposed curvelet based method gives better result with smooth character boundaries. The curvelet based method is tested for extreme noise with $\sigma=100$. The part of noisy input image at $\sigma=100$ 
and full output binary image using curvelet based method are shown in figure 7 .

Table 2: Quality comparison of final binarized document images at noise strength $\sigma=60$

\begin{tabular}{|c|c|}
\hline $\begin{array}{l}\text { Washington, } \\
\text { Pricing its IPC } \\
\text { Facebook, the } \\
\text { social networl } \\
\text { over } 45 \text { milliol }\end{array}$ & 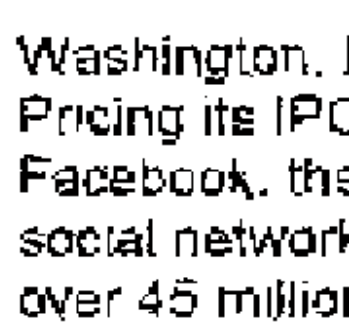 \\
\hline Original Binary Image & O/P Image of Method1 \\
\hline $\begin{array}{l}\text { Washington. } \\
\text { Pricing its IPC } \\
\text { Facebook, the } \\
\text { socjal neturot } \\
\text { over } 45 \text { milliol }\end{array}$ & $\begin{array}{l}\text { Washington, } \\
\text { Pricing its IP( } \\
\text { Facebook, thi } \\
\text { social networl } \\
\text { over } 45 \text { millio }\end{array}$ \\
\hline & \\
\hline
\end{tabular}

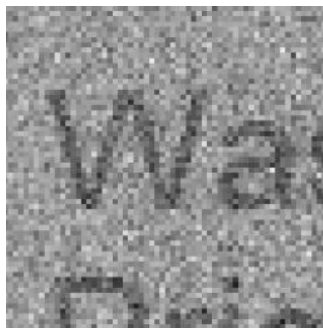

(a)
Washington, May t8 (IANS)

Pricing its $\mid P O$ at $\$ 38$ a share, Facebook, the highly popular social networking site that has over 45 miltion users in India, is expected to raise $\$ 16$ billion, making it the largest tech IPO in history.

It's reported to be the third largest US IPO ever, trailing only the $\$ 19.7$ billion raised by Visa in March 2008 and the $\$ 18.1$ billion raised by automaker General

(b)

Figure 7: (a) Part of noisy Image at $\sigma=100$, (b) Binarized output of Curvelet based method.

As can been seen from figure 7 (a), at very high level of noise, the characters are almost dissolved in background, even in such case, the curvelet based method give good results as shown in figure 7 (b). The results of other images are shown for various noise strengths from tables 3 to 5 .

Table 3: Comparison of all the three methods at different noise strengths for figure6(a)

\begin{tabular}{|c|l|c|c|c|c|}
\hline \multicolumn{2}{|c|}{ Sigma $(\boldsymbol{\sigma})$} & $\mathbf{3 0}$ & $\mathbf{4 0}$ & $\mathbf{5 0}$ & $\mathbf{6 0}$ \\
\hline \multirow{3}{*}{ Method } & FM & 92.74 & 87.69 & 81.92 & 77.20 \\
1 & NRM & 0.042 & 0.065 & 0.091 & 0.115 \\
& PSNR & 16.53 & 14.15 & 12.35 & 11.27 \\
& NC & 0.914 & 0.855 & 0.786 & 0.730 \\
\hline \multirow{3}{*}{ Method } & FM & 94.61 & 91.17 & 87.38 & 83.56 \\
2 & NRM & 0.030 & 0.046 & 0.065 & 0.080 \\
& PSNR & 17.81 & 15.62 & 14.01 & 12.76 \\
& NC & 0.936 & 0.896 & 0.851 & 0.806 \\
\hline \multirow{3}{*}{ Method } & FM & 96.88 & 95.68 & 94.62 & 93.52 \\
3 & NRM & 0.015 & 0.019 & 0.025 & 0.030 \\
& PSNR & 20.17 & 18.73 & 17.76 & 16.94 \\
& NC & 0.963 & 0.949 & 0.937 & 0.924 \\
\hline
\end{tabular}

Table 4: Comparison of all the three methods at different noise strengths for figure $6(\mathrm{c})$

\begin{tabular}{|c|l|c|c|c|c|}
\hline \multicolumn{2}{|c|}{ Sigma $(\boldsymbol{\sigma})$} & $\mathbf{3 0}$ & $\mathbf{4 0}$ & $\mathbf{5 0}$ & $\mathbf{6 0}$ \\
\hline \multirow{3}{*}{ Method } & FM & 93.84 & 90.74 & 88.43 & 86.29 \\
1 & NRM & 0.034 & 0.051 & 0.064 & 0.076 \\
& PSNR & 18.26 & 16.47 & 15.50 & 14.77 \\
& NC & 0.930 & 0.895 & 0.868 & 0.844 \\
\hline \multirow{3}{*}{ Method } & FM & 95.76 & 93.52 & 91.56 & 89.36 \\
2 & NRM & 0.024 & 0.032 & 0.046 & 0.057 \\
& PSNR & 19.88 & 18.03 & 16.87 & 15.85 \\
& NC & 0.952 & 0.926 & 0.904 & 0.879 \\
\hline \multirow{3}{*}{ Method } & FM & 98.35 & 97.66 & 96.96 & 96.16 \\
3 & NRM & 0.009 & 0.012 & 0.015 & 0.019 \\
& PSNR & 23.96 & 22.44 & 21.31 & 20.28 \\
& NC & 0.981 & 0.973 & 0.965 & 0.956 \\
\hline
\end{tabular}

Table 5: Comparison of all the three methods at different noise strengths for figure $6(d)$

\begin{tabular}{|c|l|c|c|c|c|}
\hline \multicolumn{2}{|c|}{ Sigma $(\boldsymbol{\sigma})$} & $\mathbf{3 0}$ & $\mathbf{4 0}$ & $\mathbf{5 0}$ & $\mathbf{6 0}$ \\
\hline \multirow{3}{*}{ Method } & FM & 93.12 & 88.77 & 84.50 & 81.54 \\
1 & NRM & 0.042 & 0.063 & 0.088 & 0.109 \\
& PSNR & 19.64 & 17.48 & 16.10 & 15.40 \\
& NC & 0.925 & 0.878 & 0.832 & 0.800 \\
\hline \multirow{4}{*}{ Method } & FM & 95.20 & 92.39 & 89.38 & 86.84 \\
2 & NRM & 0.030 & 0.044 & 0.059 & 0.071 \\
& PSNR & 21.19 & 19.18 & 17.72 & 16.77 \\
Method & NC & 0.948 & 0.917 & 0.885 & 0.857 \\
3 & FM & 97.46 & 96.66 & 95.77 & 94.82 \\
& NRM & 0.011 & 0.014 & 0.017 & 0.020 \\
& PSNR & 23.89 & 22.69 & 21.64 & 20.74 \\
& NC & 0.972 & 0.964 & 0.954 & 0.944 \\
\hline
\end{tabular}

As discussed in section 3.4, part of the document image of figure 6 (b) is considered to show the strength of curvelet based approach of removing Impulse noise and combination of Gaussian and Impulse noise in one processing step. These results are shown in table 6 . To remove only impulse noise, the eqn. 3 of finding threshold becomes only $\lambda_{j}=$ $K . \sqrt{2 \log \left(N_{j}\right)}$ as there is no Gaussian noise so $\sigma$ is omitted. The value of $K$ chosen is 25 .

As can be seen from the table 6 , that curvelet based method is also able to remove Impulse noise and its combination with Gaussian noise. This makes it more versatile approach to choose for denoising of document images for OCR applications. The only wavelets can also do it but a large threshold value is required. This large threshold value corrupts quality of output image too making it unsuitable for OCR purpose.

Table 6: Impulse and Gaussian noise removal using Curvelet based approach

\begin{tabular}{|c|c|}
\hline $\begin{array}{l}\text { Pricing its IPO } \\
\text { Facebook, the } \\
\text { social networki } \\
\text { over } 45 \text { million } \\
\text { expected to rais } \\
\text { making it the la }\end{array}$ & $\begin{array}{l}\text { Pricing its IPO } \\
\text { Facebook, the } \\
\text { social networki } \\
\text { over } 45 \text { million } \\
\text { expected to rai: } \\
\text { making it the la }\end{array}$ \\
\hline $\begin{array}{l}\text { Image corrupted by Impulse } \\
\text { Noise only }\end{array}$ & $\begin{array}{l}\text { Output Binary Image of } \\
\text { Method } 3(\mathrm{NC}=0.9670)\end{array}$ \\
\hline
\end{tabular}




\begin{tabular}{|c|c|}
\hline $\begin{array}{l}\text { Pricing its IPO } \\
\text { Facebook, the } \\
\text { social networki } \\
\text { over } 45 \text { million } \\
\text { expected to rai: } \\
\text { making it the le }\end{array}$ & $\begin{array}{l}\text { Pricing its IPO } \\
\text { Facebook, the } \\
\text { social networki } \\
\text { over } 45 \text { million } \\
\text { expected to rai: } \\
\text { making it the la }\end{array}$ \\
\hline $\begin{array}{c}\text { Image corrupted by Impulse } \\
\text { Noise }+ \text { Gaussian Noise } \\
(\sigma=50)\end{array}$ & $\begin{array}{l}\text { Output Binary Image of } \\
\text { Method 3(NC=0.9553) }\end{array}$ \\
\hline
\end{tabular}

To observe the performance of the proposed curvelet based scheme in OCR applications, the denoised and binarized output images are given to ABBYY Fine Reader [16] and the result of character recognition is compared with output of Fine Reader with noisy image as input. The results of character recognition are shown in table 7.

From the table 7, it is clear that the ABBYY Fine Reader is not able to recognize the text at noise standard deviation of 100. For such a noisy image, it does not recognize the whole text but with some manual adjustment, it finally recognizes some text which is shown in the table. While the output of Fine Reader with input image after using the proposed method for denoising shows very few errors. At $\sigma=75$, the Fine Reader still gives some error and there is no error visible for input image of proposed method. These results show the strength and utility of proposed curvelet based method under white Gaussian noise, impulse noise and combination of both with better OCR results.

\section{CONCLUSIONS}

Noise present in the document images always create problem for correct character recognition thus degrades the performance of any OCR system. Before further processing for OCR, this noise has to be removed effectively to get better results in character recognition. In document images, the impulse noise is found commonly because when grayscale images are binarized several black dots are visible due to uneven intensities. Also when images are transmitted over a communication channel (wirelessly), the white Gaussian noise is the main problem, for example capturing a video frame from a noisy TV signal for OCR purpose. In this paper, three different techniques of denoising are implemented and compared. The first two methods use wavelets while third method is based on curvelets with hard thresholding and modified universal threshold. The use of curvelets for denoising gives advantages of preserving edge features of characters of noisy document images which makes it suitable for OCR applications. All the three methods are compared for various types of document images of different size, font and font size. The results of section 4 show that curvelet based method is very efficient and able to preserve edge features of text upto $\sigma=100$ and even more. Also the proposed method has the strength of removing impulse noise, and combination of Gaussian and Impulse noise. The experiments with ABBYY Fine Reader shows that at very high level of noise, denoising with the proposed method is necessary to get good recognition. Without this this step, ABBYY Fine reader does not perform well.
Table 7: OCR results comparison

\begin{tabular}{|c|c|}
\hline Fine Reader I/P & Fine Reader's O/P \\
\hline $\begin{array}{l}\text { Washington, May } 18 \text { (ANS) } \\
\text { Pricing Its IPO at } \$ 38 \text { a shate, } \\
\text { Facebook, the highly popular } \\
\text { social networking site that has } \\
\text { over } 45 \text { million users in India, is } \\
\text { expected to raise } \$ 16 \text { billion, } \\
\text { making it the largest tech IPO in } \\
\text { history. } \\
\text { It's reported to be the third largest } \\
\text { US IPO ever, trailing only the } \\
\$ 19.7 \text { billion raised by Visa in } \\
\text { March } 2008 \text { and the } \$ 18.1 \text { billion } \\
\text { raised by automaker General }\end{array}$ & \multirow[t]{2}{*}{ 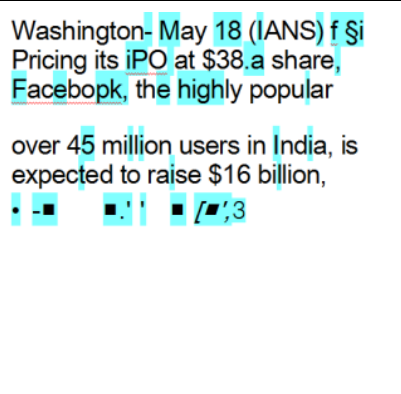 } \\
\hline Noisy Image at $\sigma=100$ & \\
\hline $\begin{array}{l}\text { Washington, May } 18 \text { (IANS) } \\
\text { Pricing its IFO at } \$ 38 \text { a share, } \\
\text { Facebook, the highly popular } \\
\text { social networking site that has } \\
\text { over } 45 \text { million users in India, is } \\
\text { expected to raise } \$ 16 \text { billion, } \\
\text { making it the largest tech IPO in } \\
\text { history. } \\
\text { It's reported to be the third largest } \\
\text { US IPO ever, trailing only the } \\
\$ 19.7 \text { billion raised by Visa in } \\
\text { March } 2008 \text { and the } \$ 18.1 \text { billion } \\
\text { raised by automaker General }\end{array}$ & \multirow[t]{2}{*}{$\begin{array}{l}\text { Washington, May } 18 \text { (IANS) } \\
\text { Pricing its IK) at } \$ 38 \text { a share, } \\
\text { FaceboQk, the highly popular } \\
\text { social networking site that has } \\
\text { over } 45 \text { million users in India, is } \\
\text { expected to raise } \$ 16 \text { billion, } \\
\text { making it the largest tech IPO in } \\
\text { history. } \\
\text { It's reported to be the third largest } \\
\text { US IPO ever trailing only the. } \\
\$ 19.7 \text { bHlion raised by Visa in } \\
\text { March } 2008 \text { and the } \$ 18.1 \text { billion } \\
\text { raised by automaker General }\end{array}$} \\
\hline $\begin{array}{l}\mathrm{O} / \mathrm{P} \text { binary Image of } \\
\text { method } 3 \text { at } \sigma=100\end{array}$ & \\
\hline $\begin{array}{l}\text { Washington, May } 18 \text { (IANS) } \\
\text { Pricing its IPO at } \$ 38 \text { a share, } \\
\text { Facebook, the highly popular } \\
\text { social networking site that has } \\
\text { over } 45 \text { million users in India, is } \\
\text { expected to raise } \$ 16 \text { billion, } \\
\text { making it the largest tech IPO in } \\
\text { history. } \\
\text { It's reported to be the third largest } \\
\text { US IPO ever, trailing only the } \\
\$ 19.7 \text { billion raised by Visa in } \\
\text { March } 2008 \text { and the } \$ 18.1 \text { billion } \\
\text { raised by automaker General }\end{array}$ & \multirow[t]{2}{*}{$\begin{array}{l}\text { Washington, May } 18 \text { (IANS) } \\
\text { Pricing its iPC at } \$ 38 \text { a share, } \\
\text { Facebook, the highly popular } \\
\text { sbcia! networking site that has } \\
\text { over } 45 \text { miiiion users in India, } \\
\text { expected to raise } \$ 16 \text { biiiion } \\
\text { making it the largest tech !PO in } \\
\text { it's reported to be the third largest } \\
\text { US IRQ } \\
\$ 19.7 \text { billion raised by Visa in ; } \\
\text { March } 2008 \text { and the } \$ 18.1 \text { biiiion } \\
\text { raised by automaker Genera! }\end{array}$} \\
\hline Noisy Image at $\sigma=75$ & \\
\hline $\begin{array}{l}\text { Washington, May } 18 \text { (IANS) } \\
\text { Pricing its IPO at } \$ 38 \text { a share, } \\
\text { Facebook, the highly popular } \\
\text { social networking site that has } \\
\text { over } 45 \text { million users in India, is } \\
\text { expected to raise } \$ 16 \text { billion, } \\
\text { making it the largest tech IPO in } \\
\text { history. } \\
\text { It's reported to be the third largest } \\
\text { US IPO ever, trailing only the } \\
\$ 19.7 \text { billion raised by Visa in } \\
\text { March } 2008 \text { and the } \$ 18.1 \text { billion } \\
\text { raised by automaker General }\end{array}$ & \multirow[t]{2}{*}{$\begin{array}{l}\text { Washington, May } 18 \text { (IANS) Pricing } \\
\text { its IPO at } \$ 38 \text { a share, Facebook, } \\
\text { the highly popular social networking } \\
\text { site that has over } 45 \text { million users in } \\
\text { India, is expected to raise } \$ 16 \\
\text { billion, making it the largest tech } \\
\text { IPO in history. } \\
\text { It's reported to be the third largest } \\
\text { US IPO ever, trailing only the } \$ 19.7 \\
\text { billion raised by Visa in March } 2008 \\
\text { and the } \$ 18.1 \text { billion raised by } \\
\text { automaker General }\end{array}$} \\
\hline $\begin{array}{l}\text { O/P binary Image of } \\
\operatorname{method} 3 \text { at } \sigma=75\end{array}$ & \\
\hline
\end{tabular}

\section{REFERENCES}

[1] Gonzalez Rafael C., Richard E. Woods, "Digital Image Processing”, 2e, PHI, ISBN 978-81-203-2758-0, 2002.

[2] Fu S., Ruan Q., Wang W. and Li Y., "Feature Preserving Nonlinear Diffusion for Ultrasonic Image Denoising and Edge Enhancement", World Academy of Science, Engineering and Technology, Vol. 2, pp. 148-151., 2005.

[3] Lei Zhang, Weisheng Dong, David Zhang, Guangming Shi, "Two-stage image denoising by principal component analysis with local pixel grouping", Elsevier Pattern Recognition, Vol. 43, Issue 4, pp. 1531-1549, 2010.

[4] Antonin Chambolle, "An Algorithm for Total Variation Minimization and Applications", Journal of Mathematical Imaging and Vision, Kluwer Academic Publishers, Vol. 20, pp. 89-97, 2004. 
[5] Leonid I. Rudin, Stanley Osher and Emad Fatemi, "Nonlinear total variation based noise removal algorithms", Elsevier Science Publishers, Physica D: Nonlinear Phenomena, Vol. 60, pp. 259-268, 1992.

[6] Ricardo D. da Silva, R. Minetto, W. R. Schwartz, H. Pedrini, "Adaptive edge-preserving image denoising using wavelet Transforms", Springer, Pattern Analysis and Applications (PAA), DOI: 10.1007/s10044-0120266-x, pp. 1-14, 2012.

[7] M. Dai,C. Peng, A. K. Chan and D. Loguinov, "Bayesian Wavelet Shrinkage with Edge Detection for SAR Image Despeckling", IEEE transactions on Geoscience and Remote Sensing, Vol. 42, No. 8, 2004.

[8] D. Gnanadurai, V. Sadasivam, "An Efficient Adaptive Thresholding Technique for Wavelet Based Image Denoising", World Academy of Science, Engineering and Technology, Vol. 1(2), pp. 114-119, 2006.

[9] J. Starck, E. J. Candes and D. L. Donoho, "The Curvelet Transform for Image Denoising", IEEE transactions on image processing, Vol. 11, No. 6, June 2002.

[10] Al-Dahoud Ali, P. D. Swami and J. Singhai, "Modified Curvelet Thresholding Algorithm for Image Denoising", Journal of Computer Science, Science Publications, Vol. 6 (1), pp. 18-23, 2010
[11] Thai V. Hoang, Elisa H. Barney Smith and Salvatore Tabbone, "Edge noise removal in bilevel graphical document images using sparse representation", Proceedings of $18^{\text {th }}$ IEEE International Conference on Image Processing (ICIP), pp.3549-3552, Brussels, Belgium, 2011

[12] Jianwei Ma, Gerlind Plonka, "Combined Curvelet Shrinkage and Nonlinear Anisotropic Diffusion”, IEEE transactions on image processing, Vol. 16, No. 9, September 2007.

[13] Linda Tessens, Aleksandra Pižurica, Alin Alecu, Adrian Munteanu and Wilfried Philips, "Context adaptive image denoising through modeling of curvelet domain statistics", SPIE Journal of Electronic Imaging, Vol. 17(3), pp. 33017-33021. 2008

[14] D. L. Donoho, "De-noising by soft thresholding”, IEEE Transaction on Information Theory, Vol. 41, pp. 613$627,1995$.

[15] I. Pratikakis, B. Gatos, K. Ntirogiannis, "Handwritten Document ImageBinarizationCompetition (HDIBCO), $12^{\text {th }}$ InternationalConferenceon frontiers in handwriting recognition, IEEE, 2010

[16] ABBYY Fine Reader: http://finereader.abbyy.com/ 